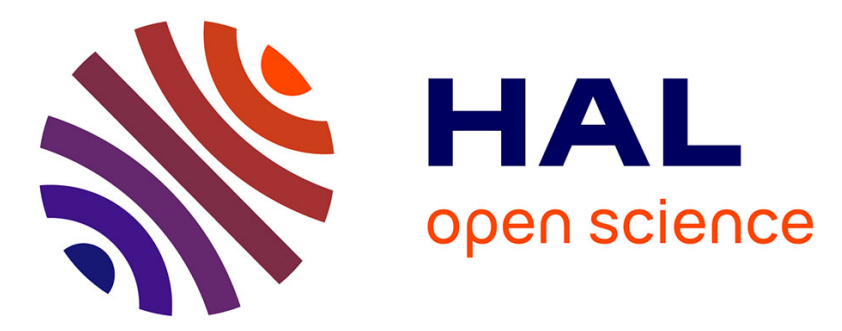

\title{
Biepitaxial Josephson junctions and SuFET technology for the preparation of HTS-JoFETs
}

K. Petersen, A. Walkenhorst, C. Stölzel, W. Wilkens, C. Krimmer, J. Söllner, H. Grueninger, R. Fischer, M. Schmitt, T. Becherer, et al.

\section{- To cite this version:}

K. Petersen, A. Walkenhorst, C. Stölzel, W. Wilkens, C. Krimmer, et al.. Biepitaxial Josephson junctions and SuFET technology for the preparation of HTS-JoFETs. Journal de Physique IV Proceedings, 1994, 04 (C6), pp.C6-205-C6-210. 10.1051/jp4:1994633 • jpa-00253128

\section{HAL Id: jpa-00253128 https://hal.science/jpa-00253128}

Submitted on 1 Jan 1994

HAL is a multi-disciplinary open access archive for the deposit and dissemination of scientific research documents, whether they are published or not. The documents may come from teaching and research institutions in France or abroad, or from public or private research centers.
L'archive ouverte pluridisciplinaire HAL, est destinée au dépôt et à la diffusion de documents scientifiques de niveau recherche, publiés ou non, émanant des établissements d'enseignement et de recherche français ou étrangers, des laboratoires publics ou privés. 


\title{
Biepitaxial Josephson junctions and SuFET technology for the preparation of HTS-JoFETs
}

\author{
K. Petersen*,**, A. Walkenhorst**, C. Stölzel*,**, W. Wilkens*, C. Krimmer*, J. Söllner*, \\ H.W. Grueninger*, R. Fischer*, M. Schmitt**, T. Becherer** and H. Adrian** \\ * Daimler-Benz Forschungsinstitut, Goldsteinstr. 235, 60528 Frankfurt/Main, Germany \\ ** Inst.f. Festk., Techn. Hochschule Darmstadt, Hochschulstr. 8, 64289 Darmstadt, Germany
}

\begin{abstract}
Biepitaxial Josephson junctions and superconducting field effect transistors. (SuFET) were fabricated in order to combine both techniques to a Josephson junction field effect transistor (JoFET). The Josephson junctions show RSJ-like behaviour. At $30 \mathrm{~K}$ a critical current density of $J_{c} \approx 10^{4} \mathrm{~A} / \mathrm{cm}^{2}$ and an $\mathrm{I}_{\mathrm{c}} \mathrm{R}_{\mathrm{N}}$-product of $0.1 \mathrm{mV}$ were obtained. The oscillation of Shapiro steps with applied microwave field can be well fitted by the RSJ-model. SuFETs with $\mathrm{CeO}_{2}$ as dielectric show, for fixed charge transfer, results comparable to $\mathrm{SrTiO}_{3}$-based SuFETs. The downset of the resistive transition of a six-unit-cells thick $\mathrm{YBa}_{2} \mathrm{Cu}_{3} \mathrm{O}_{7-\delta}$ layer is $44 \mathrm{~K}$ and the largest field effect obtained so far is $1.5 \%$. Prelimary results on JoFETs show a drastic change in the critical current density and in the specific junction resistance, however the $I_{c} R_{N^{*}}$ product remains still in the typical range. The insulating properties are degraded compared to SuFETs.
\end{abstract}

\section{Introduction}

The influence of an electric field on the transport properties of an ultrathin high temperature superconducting film was investigated by various research groups [1] [2] [3][4]. The charge carrier density can be modulated by applying a voltage from a metal gate across a dielectric material to the ultrathin $\mathrm{YBa}_{2} \mathrm{Cu}_{3} \mathrm{O}_{7-\delta}$ film. The largest field effects in SuFETs obtained so far have a modulation of charge carrier density in the range of ten percent [5]. Due to the smallness of the field effect an application of a SuFET is unlikely.

Recently, the influence of an applied electric field on ultrathin $\mathrm{YBa}_{2} \mathrm{Cu}_{3} \mathrm{O}_{7-\delta}$ films containing weak links was reported [6]. The weak links were induced by a substrate with submicron grooves. Only moderate effects were obtained in the normal conducting regime. But in the superconducting regime the field' effect was very large. So a shift in $\mathrm{T}_{c}$ of up to $25-30 \mathrm{~K}$ was observed. For a $45^{\circ}$ grain boundary Josephson junction on a bi-crystal substrate an enhancement of the Josephson supercurrent up to $70 \%$ at positive voltage was obtained [7]. This is in contrast to results obtained at SuFETs, which show an enhancement of the critical current density at negative voltage.

A JoFET is not only of interest for studying the physics of grain boundary Josephson junctions. The application of a tunable Josephson junction in high- $T_{c}$ SQUIDs opens up the possibility to reduce the difference in critical current of the two junctions [8]. In order to realize such a device it is necessary to have a planar-Josephson-junction technology as well as a SuFET technology with high quality interfaces between dielectric and superconductor. Considering these items we have decided to use biepitaxial Josephson junctions, first prepared by Char et al [9]. 


\section{Biepitaxial Josephson junctions}

\subsection{Preparation}

Biepitaxial Josephson junctions have been prepared on $\mathrm{MgO}$ substrates. It is a well known fact that $\mathrm{MgO}$ substrates become contaminated in air. In order to clean the surface the $\mathrm{MgO}$ substrates are cleaned in an ultrasonic bath of ethanol. Before the deposition, the substrates are annealed in 700 mbar $\mathrm{O}_{2}$ at $800^{\circ} \mathrm{C}$. In order to further improve the crystalline quality, a thin $\mathrm{MgO}$ layer $(\approx 10 \mathrm{~nm})$ is deposited at $590^{\circ} \mathrm{C}$ by on-axis $\mathrm{RF}$ sputtering. In the same atmosphere of $8 * 10^{-2} \mathrm{mbar} \mathrm{Ar}$ and $6 * 10^{-2}$ mbar $\mathrm{O}_{2}$ a thin $\mathrm{CeO}_{2}$ layer $(\approx 20 \mathrm{~nm})$ is sputtered in situ at $560^{\circ} \mathrm{C}$. Then on one part of the substrate the $\mathrm{CeO}_{2}$ layer is removed by ion beam etching $\left(\mathrm{U}_{\text {Bearn }}=500 \mathrm{~V}, \mathrm{~J}_{\text {Beam }}=0.5 \mathrm{~mA} / \mathrm{cm}^{2}\right)$, so that the substrate is only partly covered with $\mathrm{CeO}_{2}$. Before $\mathrm{YBa}_{2} \mathrm{Cu}_{3} \mathrm{O}_{7-\delta}$ is deposited by $\mathrm{DC}$ sputtering using an inverted cylindrical magnetron [10] in a mixed atmosphere of $4 * 10^{-1} \mathrm{mbar}$ Ar and $2 * 10^{-1}$ mbar $\mathrm{O}_{2}$, an annealing step in $700 \mathrm{mbar}_{2}$ at $800^{\circ} \mathrm{C}$ is necessary to improve the crystalline quality on the etched part of the substrate. X-ray diffraction measurements show the desired orientation $\mathrm{YBa}_{2} \mathrm{Cu}_{3} \mathrm{O}_{7-\delta}[110]\left\|\mathrm{CeO}_{2}[100]\right\| \mathrm{MgO}[100]$ on the one part of the substrate and $\mathrm{YBa}_{2} \mathrm{Cu}_{3} \mathrm{O}_{7-\delta}[100] \| \mathrm{MgO}[100]$ on the other part. To pattern the single junctions and SQUIDs, standard photolithographic methods with dilute phosphoric acid are used.

\subsection{Electrical Properties}

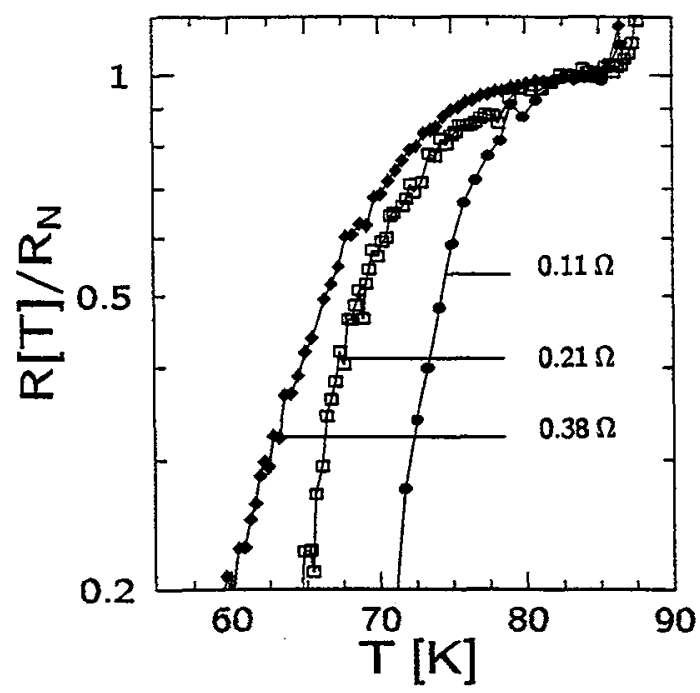

Fig.1a

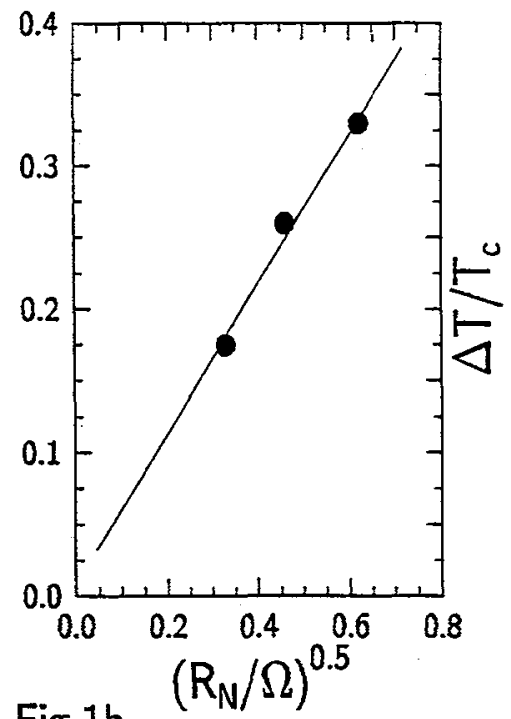

Fig.1b

Fig: 1 (a) Resistive transition of three junctions with different width (20 $\mu \mathrm{m}$ $(0.38 \Omega), 40 \mu \mathrm{m}(0.21 \Omega)$ and $60 \mu \mathrm{m}(0.11 \Omega))$. The resistance values are normalized to the junction resistances $R_{N}$. (b) There is a linear relation between the width of resistive transition and the square root of the normal resistance (see text).

The resistive transition of three different junctions on the same chip is shown in Fig. 1a. The resistance values are normalized to the normal resistance $R_{N}$ for each junction. $R_{N}$ is the resistance close to the transition of the superconducting electrodes and coincides with the junction resistance at low temperature, as is typical for grain boundary junctions [11]. The resistive transition of the 
junctions shows a foot-like structure due to thermally activated phase slippage (TAPS) [11]. Here the values for $R_{N}$ of the three junctions $(20 \mu \mathrm{m}, 40 \mu \mathrm{m}$ and $60 \mu \mathrm{m}$ width) are $0.38 \Omega, 0.21 \Omega$ and $0.11 \Omega$. Gross et al.[11] predicted for grain boundary Josephson junctions that for any fixed value $R / R_{N}$ the width of the resistive transition $\Delta T / T_{c}$ should vary with the normal resistance $R_{N}$ in the following way : $\Delta T / T_{c} \propto \sqrt{R_{N}} k_{B} T_{c} / \Delta_{i}(0)$. This analysis is based on the Ambegaokar-Bartoff expression for $I_{c}$ with a reduced energy gap $\Delta_{i}(T) \propto \Delta_{0}(T) \times\left(1-T / T_{c}\right)^{1 / 2}$ at the interface. According to Deutscher and Müller the energy gap at a superconductor-insulator interface can be reduced due to the short coherence length of the high $T_{c}$ materials [12]. In Fig. $1 b \Delta T / T_{c}$ is plotted versus $\sqrt{R_{N}}$ for a fixed value $R / R_{N}=0.2$. The good agreement between theory and experiment is shown by the plotted line, extrapolating to the origin of coordinates.

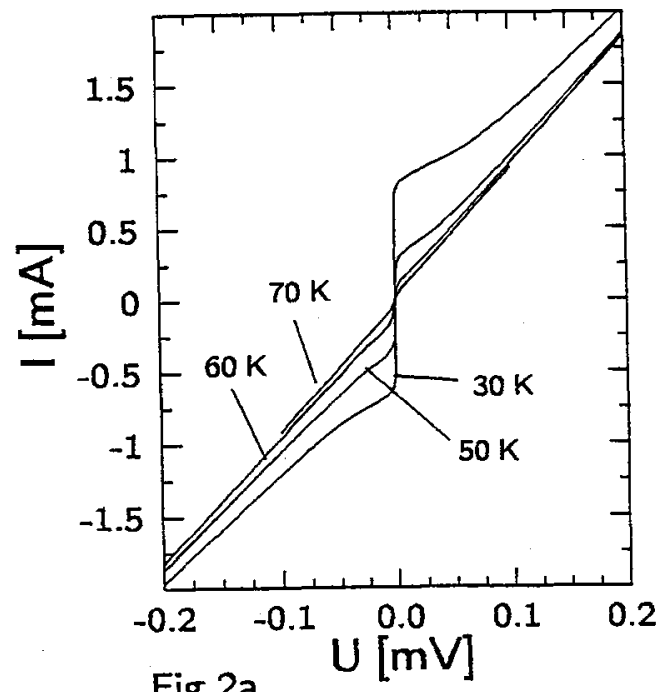

Fig.2a

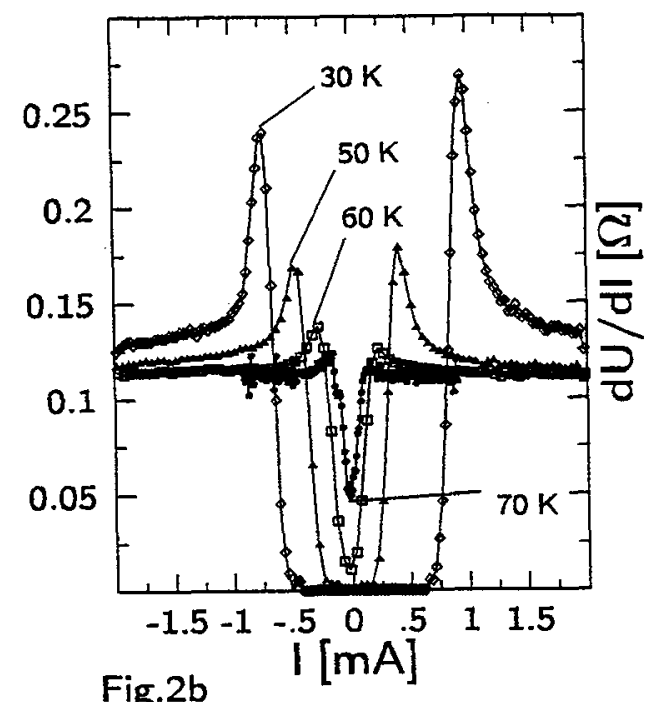

Fig. $2 b$

Fig. 2 (a) I-V curves and (b) differential resistance of a $60 \mu \mathrm{m}\left(\mathrm{R}_{\mathrm{N}}=0.11 \Omega\right)$ wide junction at $30,50,60$ and $70 \mathrm{~K}$ showing RSJ-like behaviour, the temperature independence of $R_{N}$, and the asymmetry of the critical current.

I-V-characteristics at various temperatures $(30,50,60$ and $70 \mathrm{~K})$ for a $60 \mu \mathrm{m}$ wide junction $\left(\mathrm{R}_{\mathrm{N}}=\right.$ $0.11 \Omega$ ) show typical RSJ-like behaviour (Fig. 2a). More cleary the difference in positive and negativ critical current can be seen in Fig. $2 b$, where the differential resistance is plotted versus current. Also the independence of $R_{N}$ on temperature can be seen. The I-V-curves were not measured in a magnetically shielded environment, so that trapped flux is believed to be the reason for the asymmetry. We want to point out that even in shielded environments we observed changes in critical current caused by probe currents on the same or other junctions nearby. In some cases, for applied magnetic fields ( $\leq 1$ Gauss) we even observed "hysteretic" behaviour. We explain this with trapped magnetic flux, because the McCumber parameter $\beta_{\mathrm{c}}(\ll 1)$ was certainly smaller than unity. Here the maximum probe current was not larger than five times the critical current. Nevertheless, these junctions show Shapiro step oscillations described by the RSJ-model [13].

\section{SuFET-technology}

Usually $\mathrm{SrTiO}_{3}$, due to its high dielectric constant and good chemical compatibility, is used in SuFETs with $\mathrm{YBa}_{2} \mathrm{Cu}_{3} \mathrm{O}_{7-\delta}$. However, this is related to a high loss factor tan $\delta$ leading to large dissipation already in the $\mathrm{kHz}$-regime [14]. Hence, we have used $\mathrm{CeO}_{2}$ as alternative dielectric 
material for the SuFET technology. The preparation is described in detail in [15] and will be summarized here only briefly.

In order to make low-resistance contacts to the ultrathin $\mathrm{YBa}_{2} \mathrm{Cu}_{3} \mathrm{O}_{7-\delta}$ layer, it is important to prepare thick $\mathrm{YBa}_{2} \mathrm{Cu}_{3} \mathrm{O}_{7-\delta}$ electrodes. Therefore, in a first step part of the $\mathrm{SrTiO}_{3}$ substrate is covered by a $\mathrm{SrTiO}_{3}$ mask when depositing $\approx 80 \mathrm{~nm} \mathrm{YBa}_{2} \mathrm{Cu}_{3} \mathrm{O}_{7-\delta}$. Then the mask is removed ex-situ and the ultrathin $\mathrm{YBa}_{2} \mathrm{Cu}_{3} \mathrm{O}_{7-\delta}$ layer and the insulator are deposited in-situ. The ultrathin $\mathrm{YBa}_{2} \mathrm{Cu}_{3} \mathrm{O}_{7-\delta}$ layer and the contact pads are deposited at $810^{\circ} \mathrm{C}$ by $\mathrm{DC}$ sputtering using an inverted cylindrical magnetron in a mixed atmosphere of $4 * 10^{-1}$ mbar Ar and $2 * 10^{-1}$ mbar $\mathrm{O}_{2}$ [10]. Then $\mathrm{CeO}_{2}$ is deposited in-situ at $580^{\circ} \mathrm{C}$ from a ceramic target by on axis $\mathrm{RF}$ magnetron sputtering in an atmosphere of $8 * 10^{-2}$ mbar $\mathrm{Ar}$ and $6 * 10^{-2}$ mbar $\mathrm{O}_{2}$.

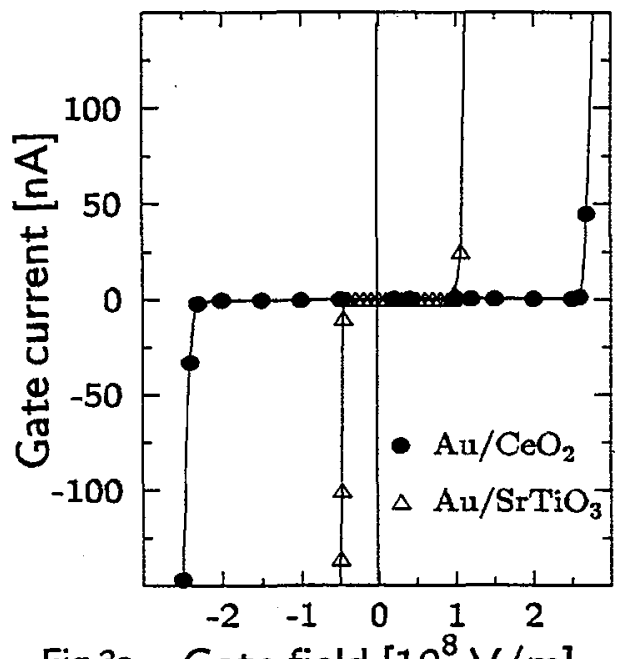

Fig.3a Gate field $\left[10^{8} \mathrm{~V} / \mathrm{m}\right]$

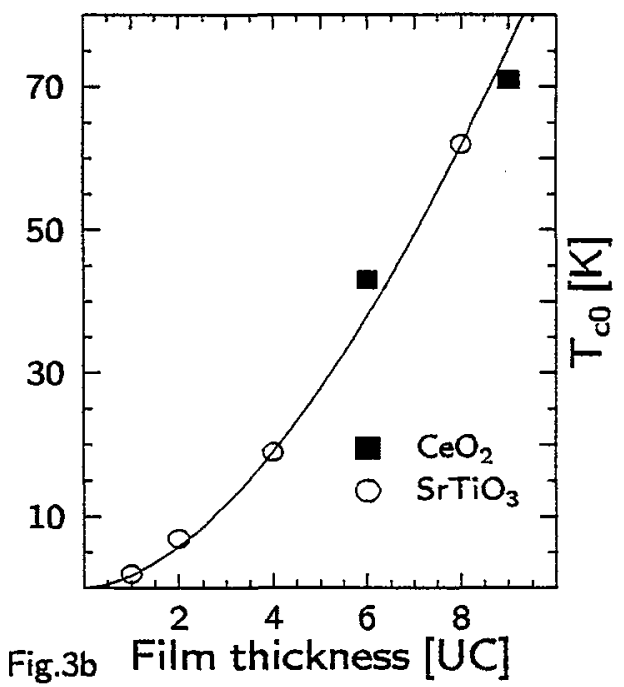

Fig.3b Film thickness [UC]

Fig. 3 (a) Symmetric breakdown characteristic of $\mathrm{CeO}_{2}$ and asymmetric characteristic of $\mathrm{SrTiO}_{3}$. (b) Dependence of downset of resistive transition on thickness of ultrathin $\mathrm{YBa}_{2} \mathrm{Cu}_{3} \mathrm{O}_{7-\delta}$ layer in SuFETs. No influence of the dielectric material can be seen.

The dielectric constant $\epsilon_{\mathrm{r}}$ of the typically $100 \mathrm{~nm}$ thick $\mathrm{CeO}_{2}$ layers is $\approx 20$ and almost temperature independent. The breakdown characteristic is symmetric, which is not the case for SuFET with $\mathrm{SrTiO}_{3}$ as dielectric material. A breakdown field of $\mathrm{E}_{\mathrm{bd}} \approx 2 \cdot 10^{8} \mathrm{~V} / \mathrm{m}$ has been achieved (Fig. 3a). The field effects for a fixed charge transfer are comparable in devices using $\mathrm{CeO}_{2}$ or $\mathrm{SrTiO}_{3}$ as dielectric, indicating that it is the change in the charge carrier density and not field-induced stresses that are responsible for the observed effects [16]. In the charge carrier enhancement mode (negative voltage) the achieved charge transfer amounts to $\Delta N=+2 \mu \mathrm{C} / \mathrm{cm}^{2}$, which is $80 \%$ of the highest value obtained for $\mathrm{SrTiO}_{3}$-based SuFETs. The surface of the ultrathin $\mathrm{YBa}_{2} \mathrm{Cu}_{3} \mathrm{O}_{7-\delta} / \mathrm{CeO}_{2}$ bilayers shows no outgrowth or pinholes in AFM studies. The average roughness of the $\mathrm{CeO}_{2}$ layer is only $3 \%$ of the total thickness. The resistive transition of a six-unit-cells thick $\mathrm{YBa}_{2} \mathrm{Cu}_{3} \mathrm{O}_{7-6}$ layer in a SuFET with $\mathrm{CeO}_{2}$ has an onset at over $80 \mathrm{~K}$ and a downset at $44 \mathrm{~K}$. This is similar to SuFETs with $\mathrm{SrTiO}_{3}$ as can be seen in Fig. 3b. The largest field effect obtained so far with the use of $\mathrm{CeO}_{2}$ as dielectric is $1.5 \%$ for a six-unit-cells thick $\mathrm{YBa}_{2} \mathrm{Cu}_{3} \mathrm{O}_{7-\delta}$ film [15]. 


\section{JoFET-technology}

To prepare a Josephson junction field effect transistor (JoFET) based on a grain boundary junction two problems have to be taken into account. First, the insulating properties of the dielectric may deteriorate when the dielectric is grown on the grain boundary. Second, the superconducting properties may change due to the preparation process of the dielectric. In addition the patterning technique may have an influence on the junction properties, when the $\mathrm{YBa}_{2} \mathrm{Cu}_{3} \mathrm{O}_{7-\delta}$ layer has to be very thin $(\leq 30 \mathrm{~nm})$ in order to achieve sizable effects. Therefore we decided to use an inhibit patterning technique as described below.

\subsection{Inhibit patterning}

After ion milling of the $\mathrm{CeO}_{2}$ seed layer the final junction area of the substrate is covered by a $\mathrm{SrTiO}_{3}$ shadow mask. Analogously to the preparation of SuFETs, contact areas of $\mathrm{YBa}_{2} \mathrm{Cu}_{3} \mathrm{O}_{7-\delta}$ are deposited at $810^{\circ} \mathrm{C}$. Then gold pads are sputtered onto the $\mathrm{YBa}_{2} \mathrm{Cu}_{3} \mathrm{O}_{7-\delta}$ contact areas through a metal shadow mask. The measurement geometry is achieved by inhibit patterning. Therefore an amorphous $\mathrm{CeO}_{2} / \mathrm{MgO} / \mathrm{CeO}_{2}$ layer system is deposited through another metal mask, covering the contact area. $\mathrm{YBa}_{2} \mathrm{Cu}_{3} \mathrm{O}_{7-\delta}$ deposited on this amorphous layer system is insulating. We choose this layer system due to its chemical compatibility to $\mathrm{YBa}_{2} \mathrm{Cu}_{3} \mathrm{O}_{7-\delta}$. In order to avoid recrystallization of the amorphous layer at the later process step at high temperatures, all three amorphous layers are necessary. In the following preparation step $\mathrm{YBa}_{2} \mathrm{Cu}_{3} \mathrm{O}_{7-\delta}$ and the insulating $\mathrm{CeO}_{2}$ are deposited in situ without any shadow mask at the conditions described for SuFETs. In the last step the Au-gate electrodes of the JoFETs are sputtered through a third metal mask.

\subsection{Prelimary results}
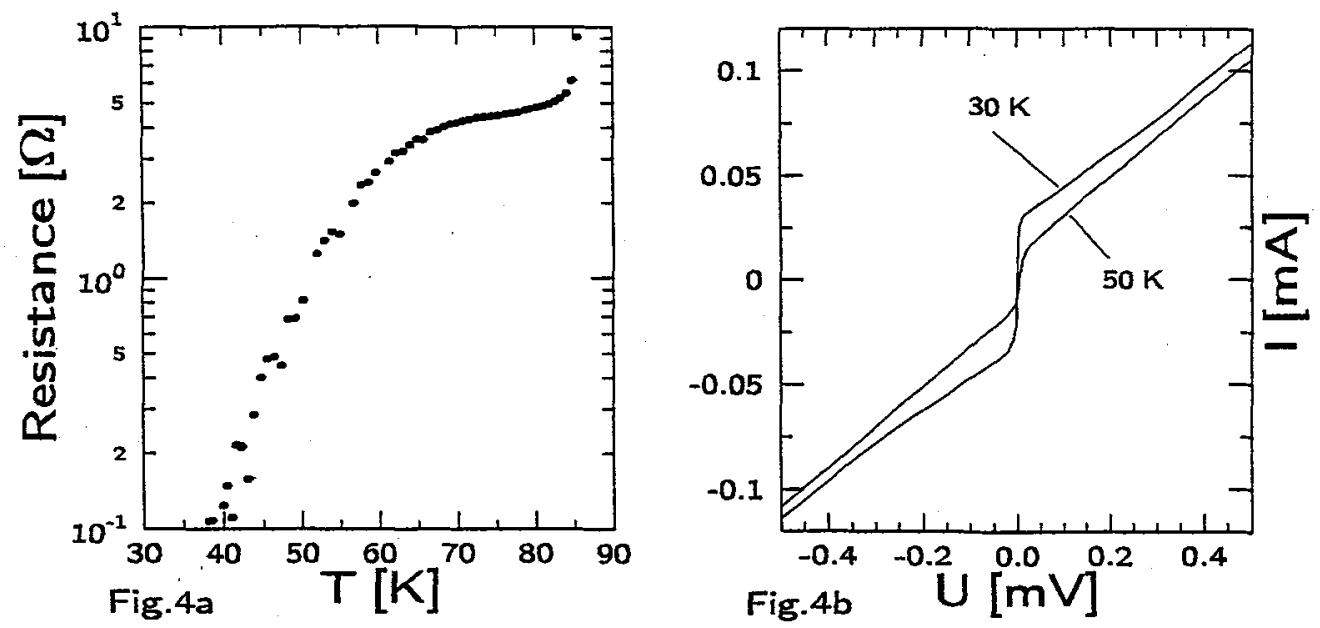

Fig. 4 (a) Resistive transition of biepitaxial Josephson junction in JoFET structure. (b) I-V curves of same junction at 30 and $50 \mathrm{~K}$.

In Fig. 4.1a the resistive transition of an inhibit patterned junction is shown. The specific normal conductance of a pure biepitaxial junction is about $50 /\left(\mu \Omega \mathrm{cm}^{2}\right)$. For a $150 \mathrm{~nm}$ thick and $200 \mu \mathrm{m}$ wide junction in the JoFET structure the conductance is more than an order of magnitude smaller. In Fig. 4.b the I-V curves for this junction are shown at 30 and $50 \mathrm{~K}$. At $30 \mathrm{~K}$ the I-V curve is still noise-rounded, so that the determination of the critical current $I_{c} \approx 25 \mu \mathrm{A}$ depends strongly on the 
$j_{c}$-criterium. The normal resistance $R_{N}=5 \Omega$ is taken from the slope of the I-V curve at higher voltages in good agreement with the foot point of the resistive transition. So the $I_{C} R_{N}$-product has a value of $0.125 \mathrm{mV}$, typical for biepitaxial junctions. Work is in progress to investigate the reason for the drastic change in critical current density and specific conductance.

The largest achieved breakdown field $E_{b d}$ in an inhibit patterned JoFET structure is $\approx 10^{7} \mathrm{~V} / \mathrm{m}$, a value one order of magnitude smaller than in SuFETs.

\section{Summary}

In summary, the preparation of biepitaxial Josephson junctions and superconducting field effect transistors has been described. The junctions are RSJ-like, and the results of the resistive transition support for high angle grain boundaries the idea of a reduced order parameter at the interface. SuFETs with $\mathrm{CeO}_{2}$ have proven to be a possible alternative to $\mathrm{SrTiO}_{3}$ based SuFETs in the charge carrier enhancement mode. The preparation of JoFETs faces the problem of changes in the junction properties. However, the $I_{c} R_{N}$-product remains unchanged. So far the insulating properties are degraded compared to SuFETs.

\section{References}

[1] J. Mannhart, J. G. Bednorz, K. A. Mueller, and D. G. Schlom, Z. Phys. B 83, 307 (1991).

[2] X. X. Xi, Q. Li, C. Doughty, C. Kwon, S. Bhattacharya, A. T. Findikoglu, and T. Venkatesan, Appl. Phys. Lett. 56, 3470 (1991).

[3] A. Jäger, J. C. Villegier, P. Bernstein, J. Bok, and L. Force, IEEE Trans. Appl. Supercond. 3, 2933 (1993).

[4] K. Joose, Yu. M. Boguslavskij, G. J. Gerritsma, and H. Rogalla, Journal of Alloys and Compounds 195, 723 (1993).

[5] X. X. Xi, C. Doughty, A. Walkenhorst, C. Kwon, Q. Li, and T. Venkatesan Phys. Rev. Lett. 68, 1241 (1992).

[6] J. Mannhart, J. Ströbel, J. G. Bednorz, and Ch. Gerber, Appl. Phys. Lett. 62, 630 (1993).

[7] Z. G. Ivanov, E. A. Stepantsov, A. Ya. Tzalenchuk, R. I. Shekhter, and T. Claeson, IEEE Trans. Appl. Supercond. 3, 2925 (1993).

[8] J. Mannhardt, European Conference on Applied Superconductivity (EUCAS'93), Göttingen, Germany, October 4-8, 1993.

[9] K. Char, M. S. Colclough, S. M. Garrison, N. Newman, and G. Zaharchuk, Appl.Phys.Lett. 59, 733 (1991).

[10] X. X. Xi, G. Linker, O. Meyer, E. Nold, B. Obst, F. Ratzel, R. Smithey, B. Strehlau, F. Weschenfelder; and J. Geerk, Z. Phys. B 74, 13 (1989).

[11] R. Gross, P. Chaudhari, D. Dimos, A. Gupta, and G. Koren, Phys. Rev. Lett. 64228 (1990).

[12] D. Deutscher and K. A. Müller, Phys. Rev. Lett. 591745 (1987).

[13] K. Petersen et al. to be published.

[14] Y. Gim, C. Doughty, X. X. Xi, A. Amar, T. Venkatesan, and F. C. Wellstood Appl. Phys. Lett. 6224 (1993).

[15] K. Petersen et al. to be published.

[16] A. Walkenhorst, K. Petersen, M. Schmitt, and H. Adrian, accepted by Appl. Phys. Lett. 